\title{
Model predictive control of a time-varying convection-diffusion equation with state constraints
}

\author{
Lars Grüne* Simon Pirkelmann** \\ * Chair of Applied Mathematics, University of Bayreuth, 95447 \\ Bayreuth, Germany (e-mail: lars.gruene@uni-bayreuth.de) \\ ** Chair of Applied Mathematics, University of Bayreuth, 95447 \\ Bayreuth, Germany (e-mail: simon.pirkelmann@uni-bayreuth.de)
}

Keywords: Time-varying systems, model predictive control, partial differential equations

\section{INTRODUCTION AND OUTLINE}

In this discussion paper we consider the convectiondiffusion equation with time-varying boundary conditions as a simple model of a room whose temperature shall be controlled in an energy efficient way. This model serves as an example for which we want to investigate whether results from a recent paper (Grüne and Pirkelmann, 2017) also hold for a more involved model.

In the first part we introduce the model and formulate a constrained optimization problem. The following section outlines how an approximate solution to the problem can be obtained using Model Predictive Control (MPC). The method is briefly described and we also mention results about the relation between the MPC solution and the solution of the original problem. The final part gives a brief overview of selected aspects of the implementation.

\section{PROBLEM STATEMENT}

Consider the heat equation

$$
\begin{gathered}
\frac{\partial y}{\partial t}-\alpha \nabla^{2} y+w \nabla y=0 \text { on } Q:=\Omega \times[0, T], \\
y(0)=y_{0} \text { on } \Omega,
\end{gathered}
$$

where $y: Q \rightarrow \mathbb{R}$ is the temperature, $\alpha>0$ is the diffusion coefficient, $w:[0, T] \times \Omega \rightarrow \mathbb{R}$ is a velocity field and $y_{0}: \Omega \rightarrow \mathbb{R}$ is the initial condition.

As a domain $\Omega$ we consider the unit interval. The boundary $\Gamma$ is partitioned into a controlled boundary $\Gamma_{c}$ and an uncontrolled boundary $\Gamma_{\text {out }}$, see Figure 1 . On the controlled part of the boundary a function $u$ is applied representing heating and cooling. This is modelled by the condition

$$
\beta \frac{\partial y}{\partial n}+\gamma_{c} y=\gamma_{c} u \text { on } \Sigma_{c}:=\Gamma_{c} \times[0, T] .
$$

On the uncontrolled part we have

$$
\beta \frac{\partial y}{\partial n}+\gamma_{\text {out }} y=\gamma_{\text {out }} y_{\text {out }} \text { on } \Sigma_{\text {out }}:=\Gamma_{\text {out }} \times[0, T] .
$$

In the above equations $\frac{\partial y}{\partial n}$ is the derivative of $y$ in normal direction, $y_{\text {out }}$ is the outside temperature, and $\beta: \Sigma \rightarrow \mathbb{R}$, $\gamma_{c}: \Sigma_{c} \rightarrow \mathbb{R}$ and $\gamma_{\text {out }}: \Sigma_{\text {out }} \rightarrow \mathbb{R}$ are coefficient functions. Defining $\gamma:=\left\{\begin{array}{ll}\gamma_{\text {out }} & \text { on } \Gamma_{\text {out }} \\ \gamma_{c} & \text { on } \Gamma_{c}\end{array}\right.$ and $z:= \begin{cases}y_{\text {out }} & \text { on } \Gamma_{\text {out }} \\ u & \text { on } \Gamma_{c}\end{cases}$

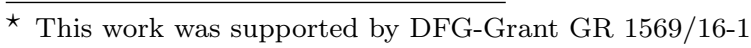

the two above boundary conditions can be written in a more consise way:

$$
-\beta \frac{\partial y}{\partial n}=\gamma(y-z) \text { on } \Sigma
$$

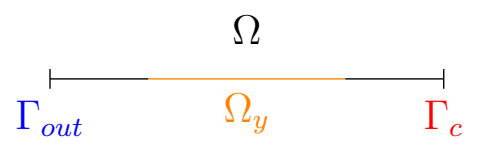

Fig. 1. Illustration of domain $\Omega$ and subdomain $\Omega_{y}$, as well as controlled $\left(\Gamma_{c}\right)$ and uncontrolled $\left(\Gamma_{\text {out }}\right)$ parts of the boundary.

In addition to controlling the temperature at the boundary we may control the velocity field $w$. For simplicity we assume that for any fixed time point $w$ is constant on the whole domain, i.e. does not depend on space.

The presented model is motivated by a practical application: energy efficient building control. We would like to influence the temperature of a room $(\Omega)$ by controlling the temperature at one wall of the room $\left(\Gamma_{c}\right)$ and the airflow inside the room. The temperature at the other wall cannot be controlled and may be changing over time (e.g. due to changing weather). However, we assume that the outside temperature $y_{\text {out }}$ is known in advance via the weather forecast, at least for a certain time span into the future.

Our goal is to keep the temperature in some part of the room $\Omega_{y}$ within certain upper and lower bounds $\underline{y}$ and $\bar{y}$, and doing so using as little control effort (or energy) $u$ and $w$ as possible. In addition, we may also want to penalize the deviation from some reference temperature $y_{\Omega}$.

This is expressed by the following optimal control problem:

$$
\begin{aligned}
\min _{y, u} J(y, u)= & \frac{\varepsilon_{y}}{2}\left\|y(T)-y_{\Omega}(T)\right\|_{L^{2}(\Omega)}^{2}+\frac{\varepsilon_{y}}{2}\left\|y-y_{\Omega}\right\|_{L^{2}(Q)}^{2} \\
& +\frac{\varepsilon_{u}}{2}\|u\|_{L^{2}(\Sigma)}^{2}+\frac{\varepsilon_{w}}{2}\|w\|_{L^{2}(0, T)}^{2}
\end{aligned}
$$

subject to equations (2), (5) and the constraints

$$
\begin{aligned}
& \underline{u} \leq u \leq \bar{u} \text { on } \Sigma_{c}, \\
& \underline{y} \leq y \leq \bar{y} \text { on } \Omega_{y} \times[0, T],
\end{aligned}
$$

with (possibly time-varying) lower and upper bounds for state and control. 


\section{MODEL PREDICTIVE CONTROL}

For large or possibly infinite time horizon $T$ the problem becomes very difficult to solve numerically. Also, reliable weather forecast data will only be available for relatively short periods. To deal with this issue Model Predictive Control (MPC) is used. For an in-depth introdution to MPC we refer to Grüne and Pannek (2017).

Briefly, the idea is as follows: Starting at time $t=0$ given the state $y(0)=y_{0}$ we consider the problem on a shorter horizon $\left[0, T_{M P C}\right]$ with $T_{M P C}<T$ and solve the reduced open-loop problem instead. As a solution we obtain the control $u$ on the smaller horizon which is then applied to the system up to $t=h$ with some sampling rate $h<T_{M P C}$. At time $t=h$ the state is measured again and the procedure is repeated. Continuing this way we obtain the closed-loop of the controlled system.

The natural question now is whether the MPC closedloop approximates the solution of the original problem. Our recent results for time-varying systems show that at least the cost of an infinite horizon optimal solution is approximated by the MPC algorithm, cf. Grüne and Pirkelmann (2017). In that paper we used a rather simple example to illustrate the results.

The model presented in the current paper is more involved and will serve to demonstrate that approximate optimality can also be observed in the infinite-dimensional setting.

\section{IMPLEMENTATION DETAILS}

MPC reduces the problem in time but we still need to solve an open-loop optimal control problem in each step of the MPC algorithm. We use a First-Discretize-Then-Optimize approach to solve the problem.

Discretizing the optimal control problem (6), (8) in time and space we obtain a finite-dimensional optimization problem with a quadratic cost functional

$$
\begin{aligned}
& \min _{y_{h}, u, w} J\left(y_{h}, u, w\right)= \\
& \sum_{k=0}^{N-1}\left(\frac{\varepsilon_{y}}{2}\left(y_{h, k}-y_{\Omega, k}\right)^{T} Q\left(y_{h, k}-y_{\Omega, k}\right)+\frac{\varepsilon_{u}}{2} u_{k}^{T} R u_{k}\right. \\
& \left.+\frac{\varepsilon_{w}}{2} w_{k}^{T} W w_{k}\right)+\frac{\varepsilon_{y}}{2}\left(y_{h, N}-y_{\Omega, N}\right)^{T} Q\left(y_{h, N}-y_{\Omega, N}\right)
\end{aligned}
$$

subject to the nonlinear constraints

$$
\begin{array}{lr}
A y_{h, k+1}+w_{k} B_{w} y_{h, k+1}=B_{y} y_{h, k}+b_{u} u_{k}+b_{y, \text { out }} y_{o u t, k} \\
\text { for } k \in\{0, \ldots, N-1\}, \\
\underline{y}_{h, k, i} \leq y_{h, k, i} \leq \bar{y}_{h, k, i} & \text { for } \left.k \in\{0, \ldots, N\}, i \in \mathcal{I}_{\Omega_{y},}, \ldots, N-1\right\}, \\
\underline{u}_{k} \leq u_{k} \leq \bar{u}_{k} & \text { for } k \in\{0, \ldots, N-1\}
\end{array}
$$

where the matrices $A, B_{w}, B_{y}$ and the vectors $b_{u}$ and $b_{y, \text { out }}$ stem from the finite element discretization and $Q, R$ and $W$ are weighting matrices and $\mathcal{I}_{\Omega_{y}}$ is an index set corresponding to finite element nodes inside the subdomain $\Omega_{y}$. The implementation is carried out using FEniCS, cf. Alnæs et al. (2015), Logg et al. (2012), for the finite element discretization and MATLAB's fmincon solver for the optimization. The source code for our implementation of the MPC algorithm can be found on GitHub, see references. Our numerical simulations show that the costs of the closed-loops seem to converge for increasing horizon lengths (see Fig. 2). In addition, it was investigated

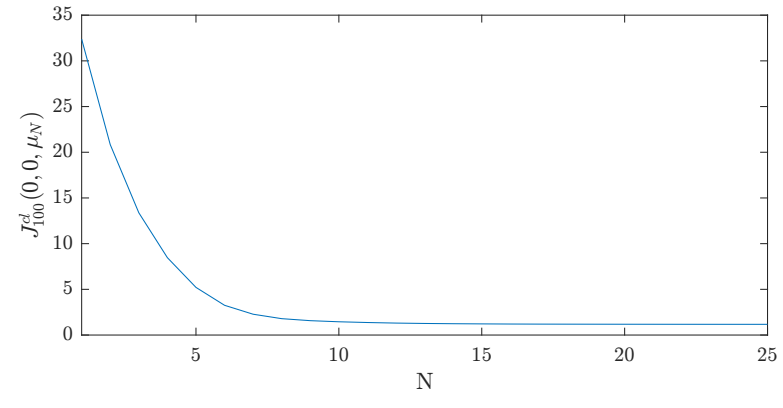

Fig. 2. Closed-loop cost of the MPC loop for a simulation over a time period of $T=1$.

whether the open-loop trajectories of the optimization have the turnpike property which is the key property of the optimal trajectories needed for proving near optimality of the MPC solutions. The corresponding numerical results will be presented in detail in the talk.

\section{OUTLOOK}

While the convergence of closed-loop costs can be explained by our theoretical results, it is not yet proven that the state and control trajectories also converge. This will be the subject of further investigations.

Another challenge is that the current implementation scales poorly with the degrees of freedom of the finite element discretization and therefore larger problems in multidimensional domains have not yet been addressed. A first approach to deal with this issue is the use of Proper Orthogonal Decomposition as described in (Mechelli and Volkwein, 2017). We are currently working with the authors on a more efficient implementation of our problem.

\section{REFERENCES}

Alnæs, M.S., Blechta, J., Hake, J., Johansson, A., Kehlet, B., Logg, A., Richardson, C., Ring, J., Rognes, M.E., and Wells, G.N. (2015). The fenics project version 1.5. Archive of Numerical Software, 3(100). doi:10.11588/ ans.2015.100.20553.

Grüne, L. and Pannek, J. (2017). Nonlinear Model Predictive Control. Theory and Algorithms. Springer, second edition.

Grüne, L. and Pirkelmann, S. (2017). Closed-loop performance analysis for economic model predictive control of time-varying systems. Proceedings of the 56th IEEE Conference on Decision and Control (CDC).

Logg, A., Mardal, K.A., Wells, G.N., et al. (2012). Automated Solution of Differential Equations by the Finite Element Method. Springer. doi:10.1007/978-3-64223099-8.

Mechelli, L. and Volkwein, S. (2017). Pod-based economic optimal control of heat-convection phenomena. Konstanzer Schriften in Mathematik 365, University of Konstanz.

Pirkelmann, S. (2018). Convection-diffusion-equation-pdecontrol. https://github.com/Telos4/convectiondiffusion-equation-fdto.

Tröltzsch, F. (2010). Optimal control of partial differential equations: theory, methods and applications. American Mathematical Society, Providence, Rhode Island. 